\title{
Groups whose non-permutable subgroups are metaquasihamiltonian
}

\author{
Maria Ferrara and Marco Trombetti \\ Communicated by Andrea Lucchini
}

\begin{abstract}
If $\mathfrak{X}$ is a class of groups, define a sequence of group classes $\mathfrak{X}_{1}, \mathfrak{X}_{2}, \ldots, \mathfrak{X}_{k}, \ldots$ by putting $\mathfrak{X}_{1}=\mathfrak{X}$ and choosing $\mathfrak{X}_{k+1}$ as the class of all groups whose non-permutable subgroups belong to $\mathfrak{X}_{k}$. In particular, if $\mathfrak{X}$ is the class of abelian groups, $\mathfrak{X}_{2}$ is the class of quasimetahamiltonian groups, i.e. groups whose non-permutable subgroups are abelian. The aim of this paper is to study the structure of $\mathfrak{X}_{k}$-groups, with special emphasis on the case $\mathfrak{X}=\mathfrak{U}$. Among other results, it will also be proved that a group has a finite normal subgroup with quasihamiltonian quotient if and only if it is locally graded and belongs to $\mathfrak{U}_{k}$ for some positive integer $k$.
\end{abstract}

\section{Introduction}

Let $G$ be a group. A subgroup $H$ of $G$ is called permutable (in $G$ ) if $H K=K H$ for all subgroups $K$ of $G$. Groups in which all proper subgroups are permutable, that is, quasihamiltonian groups, have been completely characterized (see [13]); thus the problem arose of understanding the structure of groups for which the set of non-permutable subgroups is small in some sense (see for instance $[2-4,6]$ ).

A group is called metaquasihamiltonian if all its non-abelian subgroups are permutable. Metaquasihamiltonian groups were introduced and studied by De Falco, de Giovanni, Musella and Schmidt in [2]. Of course, Tarski groups (that is, infinite simple groups whose proper non-trivial subgroups have prime order) are metaquasihamiltonian so that, in order to avoid pathological cases of this type, it is usual to work within the large universe of locally graded groups; here a group $G$ is said to be locally graded if every finitely generated non-trivial subgroup of $G$ contains a proper subgroup of finite index. Under this assumption, it was proved that metaquasihamiltonian groups are finite-by-quasihamiltonian, i.e., they have a finite normal subgroup with quasihamiltonian quotient. In particular, locally graded torsion-free metaquasihamiltonian groups are abelian.

The authors are supported by GNSAGA (INdAM), and are members of the no-profit association AGTA - Advances in Group Theory and Applications (www . advgrouptheory . com). 
The aim of this paper is to provide a further contribution to this topic by looking at metaquasihamiltonian groups in the general framework of group classes that can be obtained by iterating a restriction on non-permutable subgroups. This approach has first been used in [7] to obtain a characterization of groups with a finite commutator subgroup (see also [8]).

Let $\mathfrak{X}$ be a class of groups. Put $\mathfrak{X}_{1}=\mathfrak{X}$, and suppose by induction that a group class $\mathfrak{X}_{k}$ has been defined for some positive integer $k$; then we denote by $\mathfrak{X}_{k+1}$ the class consisting of all groups in which every non-permutable subgroup belongs to $\mathfrak{X}_{k}$. Of course, a group class $\mathfrak{X}$ is not in general contained in $\mathfrak{X}_{2}$, as the consideration of the class of simple groups shows (see also [13, Theorem 6.4.1]), but this is certainly the case if $\mathfrak{X}$ is closed with respect to forming subgroups. Moreover, for each positive integer $k$, the class $\mathfrak{X}_{k+1}$ is subgroup closed, and $\mathfrak{X}_{k+1}=\left(\mathfrak{X}_{k}\right)_{2}$ so that $\mathfrak{X}_{h} \leq \mathfrak{X}_{k}$ whenever $2 \leq h \leq k$, and we put

$$
\mathfrak{X}_{\infty}=\bigcup_{k \geq 1} \mathfrak{X}_{k}
$$

A similar construction was carried out in [7], where normality replaced permutability; the class corresponding to $\mathfrak{X}_{k}$ in that work will be here denoted by $\overline{\mathfrak{X}}_{k}$ for all $k$ in $\mathbb{N} \cup\{\infty\}$; clearly, in general, $\mathfrak{X}_{1}=\overline{\mathfrak{X}}_{1}=\mathfrak{X}$ and $\overline{\mathfrak{X}}_{i} \subseteq \mathfrak{X}_{i}$ for all $i \geq 2$.

If $\mathfrak{X}$ is chosen to be the class $\mathfrak{A}$ of abelian groups, we have that $\mathfrak{A}_{2}$ is the usual class of metaquasihamiltonian groups and $\mathfrak{A}_{3}$ consists of all groups whose nonpermutable subgroups are metaquasihamiltonian. Members of the class $\mathfrak{A}_{k}$ will be called $k$-quasihamiltonian groups (for any $k$ ). Thus 1-quasihamiltonian and 2-quasihamiltonian groups are precisely the abelian and the metaquasihamiltonian groups, respectively. In this paper, we will investigate the structure of $k$-quasihamiltonian locally graded groups for $k \geq 3$.

Let $k$ be any positive integer, and let $p_{1}, \ldots, p_{k}$ be pairwise distinct odd prime numbers. If $A$ is a cyclic group of order $p_{1} \cdot \ldots \cdot p_{k}$ and $x$ is the automorphism of $A$ which inverts all elements, the semidirect product $E_{k}=\langle x\rangle \ltimes A$ is a $(k+1)$ quasihamiltonian group which is not $k$-quasihamiltonian. Hence $\mathfrak{A}_{k}$ is a proper subclass of $\mathfrak{A}_{k+1}$ for each positive integer $k$. If $H_{k, q}$ is a quasihamiltonian $q$-group with infinite commutator subgroup for a prime $q \neq p_{1}, \ldots, p_{k}$, then $E_{k} \times H_{k, q}$ is in the same conditions as above, plus it is not finite-by-abelian, i.e., it has infinite commutator subgroup; in particular, $\overline{\mathfrak{A}}_{k}$ is strictly contained in $\mathfrak{A}_{k}$.

It is clear that $\mathfrak{X}_{k} \leq \mathfrak{Y}_{k}$ for all $k$ whenever $\mathfrak{X}$ and $\mathfrak{Y}$ are arbitrary group classes such that $\mathfrak{X} \leq \mathfrak{Y}$. Notice also that if $\mathfrak{Q}$ is the class of quasihamiltonian groups, then the classes $\mathfrak{A}_{k}$ and $\mathfrak{Q}_{k}$ do not coincide as the consideration of the direct product of $k$ copies of the quaternion group of order 8 shows. However, it should be noticed that $\mathfrak{\bigotimes}_{k} \subseteq \mathfrak{U}_{k+1}$ for all positive integers $k$, which implies that $\mathfrak{Q}_{\infty}=\mathfrak{U}_{\infty}$; it is perhaps unnecessary to make the reader notice that a direct product of $k$ 
coprime infinite extraspecial groups of prime exponent shows that the inclusion $\mathfrak{Q}_{k} \subseteq \mathfrak{A}_{k+1}$ is strict.

The main result of the paper provides a characterization of the class $\mathfrak{F}\{$ consisting of all finite-by-quasihamiltonian groups; in fact, we will show that we have $\mathfrak{F} \mathfrak{Q}=\mathfrak{A}_{\infty}$ in the universe of locally graded groups. Therefore, the class $\mathfrak{F} \mathfrak{Z}$ is saturated by those of $k$-quasihamiltonian locally graded groups when $k$ ranges over all positive integers.

Most of our notation is standard and can be found in [12]. For a full account of permutable subgroups and quasihamiltonian groups, we refer to [13].

\section{Statements and proofs}

As we pointed out in the introduction, the class $\mathfrak{X}_{k}$ is closed with respect to subgroups for every $k>1$, even if the class $\mathfrak{X}=\mathfrak{X}_{1}$ is not subgroup closed. We will begin this section by considering some closure properties that are inherited from $\mathfrak{X}$ to the group classes $\mathfrak{X}_{k}$.

First of all, notice that if $\mathfrak{X}$ is a group class which is closed with respect to homomorphic images, the same property obviously holds also for every $\mathfrak{X}_{k}$. Recall that a group class $\mathfrak{X}$ is local if a group $G$ belongs to $\mathfrak{X}$ whenever each of its finite subsets is contained in an $\mathfrak{X}$-subgroup. Clearly, a subgroup closed group class $\mathfrak{X}$ is local if and only if it contains all groups whose finitely generated subgroups belong to $\mathfrak{X}$. Our first lemma shows that the property of being local is inherited by the classes $\mathfrak{X}_{k}$.

Lemma 2.1. Let $\mathfrak{X}$ be a local group class. Then, for each positive integer $k$, the class $\mathfrak{X}_{k}$ is also local.

Proof. Since $\mathfrak{X}_{1}=\mathfrak{X}$, the statement is obvious if $k=1$. Suppose now that the class $\mathfrak{X}_{k}$ is local for some positive integer $k$. As $\mathfrak{X}_{k+1}$ is subgroup closed, it is enough to prove that a group $G$ belongs to $\mathfrak{X}_{k+1}$ provided that all its finitely generated subgroups are $\mathfrak{X}_{k+1}$-groups. Let $X$ be any subgroup of $G$ which is not in $\mathfrak{X}_{k}$, and let $\mathcal{W}_{X}$ be the set of all finitely generated subgroups of $X$ which are not contained in an $\mathfrak{X}_{k}$-subgroup of $X$. Then $\mathfrak{W}_{X}$ is not empty because $\mathfrak{X}_{k}$ is local. If $g$ is any element of $G$ and $U \in \mathcal{W}_{X}$, the subgroup $\langle g, U\rangle$ belongs to $\mathfrak{X}_{k+1}$, whence $\langle g\rangle U=U\langle g\rangle$. It follows that all elements of $\mathcal{W}_{X}$ are permutable in $G$. Moreover, $\langle x, U\rangle \in \mathcal{W}_{X}$ for all $x \in X$, and so $X=\left\langle V \mid V \in \mathcal{W}_{X}\right\rangle$ is likewise permutable in $G$. Therefore, $G$ belongs to $\mathfrak{X}_{k+1}$, and hence $\mathfrak{X}_{k+1}$ is a local class.

Since the class of abelian groups is obviously local, the choice $\mathfrak{X}=\mathfrak{U}$ in the above statement gives of course the following interesting special case. 
Corollary 2.2. For each positive integer $k$, the class of $k$-quasihamiltonian groups is local.

The following result shows in particular that locally graded $k$-quasihamiltonian groups are locally (soluble-by-finite), i.e., every finitely generated subgroup has a soluble subgroup of finite index.

Lemma 2.3. Let $\mathfrak{X}$ be a class of locally (soluble-by-finite) groups. Then all locally graded, finitely generated $\mathfrak{X}_{k}$-groups are soluble-by-finite for all $k \geq 1$.

Proof. Working by induction on $k$, we may assume $k \geq 2$. Let $G$ be a locally graded, finitely generated $\mathfrak{X}_{k}$-group. If all proper subgroups of $G^{\prime \prime}$ have the $\mathfrak{X}_{k-1^{-}}$ property, then $G^{\prime \prime}$ is either $\mathfrak{X}_{k-1}$ itself or finitely generated (see Lemma 2.1). In any case, being locally graded, it is soluble-by-finite by the induction hypothesis and hence even $G$ is such.

By Lemma 2.1, it is now possible to take a finitely generated proper subgroup $X$ of $G^{\prime \prime}$ which is not $\mathfrak{X}_{k-1}$ (use Lemma 2.1). Then $X$ is permutable in $G$, and so $\left|X^{G}: X\right|$ is finite. Clearly, $G / X^{G}$ is a quasihamiltonian group so that $G^{\prime \prime}=X^{G}$ for all such subgroups $X$; in particular, $G^{\prime \prime \prime}=G^{\prime \prime}$, and $G^{\prime \prime}$ is perfect. Thus the finite group $X^{G} / X_{X^{G}}$ cannot be non-trivial soluble, and hence $X_{X^{G}}$ has the $\mathfrak{X}_{k-1}$-property. By induction, it is soluble-by-finite, along with $G^{\prime \prime}$ and $G$.

Before going on to the next result, we need to recall some definitions given in [7]. Let $\mathfrak{X}$ be a group class. A subgroup $X$ of a group $G$ is said to be compressed by $\mathfrak{X}$ if it contains a normal subgroup $N$ of $G$ such that $G / N$ is an $\mathfrak{X}$-group; in this case, such a subgroup $N$ will be called an $\mathfrak{X}$-compressor for $X$ in $G$. Of course, if the class $\mathfrak{X}$ is closed with respect to homomorphic images, the core $X_{G}$ of an $\mathfrak{X}$-compressed subgroup $X$ is an $\mathfrak{X}$-compressor for $X$ in $G$. It is also clear that, in any group, the class of finite groups compresses all subgroups of finite index.

Lemma 2.4. Let $\mathfrak{X}$ be a group class, and let $X, Y$ be subgroups of a group $G$ such that $X$ is not permutable in $G$ and $Y \leq X$. If $Y$ is compressed in $G$ by the class $\mathfrak{X}_{k}$ for some integer $k>1$, then $Y$ is compressed in $X$ by $\mathfrak{X}_{k-1}$.

Proof. Let $N$ be an $\mathfrak{X}_{k}$-compressor for $Y$ in $G$. Then $X / N$ is a non-permutable subgroup of the $\mathfrak{X}_{k}$-group $G / N$, and hence it belongs to $\mathfrak{X}_{k-1}$, which means that $Y$ is compressed by $\mathfrak{X}_{k-1}$ in $X$.

We also need to recall the definition of a Robinson class. We shall say that a subgroup closed group class $\mathfrak{X}$ is a Robinson class if every finitely generated hyper(abelian or finite) group, whose subgroups of finite index are compressed by $\mathfrak{X}$, belongs to $\mathfrak{X}$ and is polycyclic-by-finite. Recall here that a group is hyper-(abelian 
or finite) if it has an ascending normal series whose factors are either abelian or finite. If the class $\mathfrak{X}$ is closed also with respect to homomorphic images, we have that $\mathfrak{X}$ is a Robinson class if and only if every finitely generated hyper-(abelian or finite) group whose finite homomorphic images belong to $\mathfrak{X}$ is polycyclic-by-finite and belongs to $\mathfrak{X}$. The most relevant group class of this type is that of nilpotent groups; a result that was proved by Robinson in [11]. On the other hand, although any polycyclic-by-finite group whose finite homomorphic images are supersoluble is likewise supersoluble (see [1]); it is easy to see that supersoluble groups do not form a Robinson class. It follows easily from Robinson's theorem and from the fact that polycyclic groups are residually finite that also the class $\mathfrak{N}_{c}$ of all nilpotent groups of class at most $c$ has the Robinson property; thus $\mathfrak{U}$ is a Robinson class. Our next theorem proves in particular that $k$-quasihamiltonian groups form a Robinson class for each positive integer $k$.

Theorem 2.5. Let $\mathfrak{X}$ be a Robinson class of groups. Then, for each positive integer $k$, also the class $\mathfrak{X}_{k}$ has the Robinson property.

Proof. The statement is obvious if $k=1$. Suppose now by induction on $k$ that $\mathfrak{X}_{k}$ is a Robinson class for some positive integer $k$, and let $G$ be any finitely generated hyper-(abelian or finite) group in which all subgroups of finite index are compressed by $\mathfrak{X}_{k+1}$. If all subgroups of finite index of $G$ are permutable, then every finite homomorphic image of $G$ is nilpotent, and so it follows from Robinson's theorem that $G$ itself is nilpotent, and hence also polycyclic. On the other hand, if $G$ contains a non-permutable subgroup $X$ of finite index, Lemma 2.4 yields that every subgroup of finite index of $X$ is compressed in $X$ by the Robinson class $\mathfrak{X}_{k}$; then, by definition, $X$ must contain a polycyclic subgroup of finite index, and so $G$ is polycyclic-by-finite in any case.

Let $H$ be a subgroup of $G$ which is not $\mathfrak{X}_{k}$. Then, by induction, there is a subgroup $K$ of finite index in $H$ that is not $\mathfrak{X}_{k}$-compressed in $H$. Now $K=H \cap L$ for some subgroup $L$ of finite index in $G$. If $N$ is any normal subgroup of finite index of $G$, then $H / H \cap L_{G} \cap N$ is not $\mathfrak{X}_{k}$, and hence $H\left(L_{G} \cap N\right) /\left(L_{G} \cap N\right)$ is permutable in $G /\left(L_{G} \cap N\right)$. Now [10, Theorem A*] shows that $H$ is permutable in $G$. This shows that $G$ is $\mathfrak{X}_{k+1}$ and completes the proof of the theorem.

Let $\mathfrak{X}$ be a class of groups. A group is said to be minimal non- $\mathfrak{X}$ if it is not an $\mathfrak{X}$-group but all its proper subgroups belong to $\mathfrak{X}$. We shall say that a group class $\mathfrak{X}$ is accessible if every locally graded group whose proper subgroups belong to $\mathfrak{X}$ is either finite or an $\mathfrak{X}$-group, or equivalently if any locally graded minimal non- $\mathfrak{X}$ group is finite. In particular, a group class $\mathfrak{X}$ containing all finite groups is accessible if and only if there are no minimal non- $\mathfrak{X}$ groups in the universe of 
locally graded groups. It is easy to show that abelian groups form an accessible group class, and $\mathfrak{A}$ shares such a property with other relevant classes of groups. Further information on accessible group classes can be found in [5].

Theorem 2.6. Let $\mathfrak{X}$ be a Robinson class made of locally (soluble-by-finite) groups which is local and closed with respect to homomorphic images. If $\mathfrak{X}$ is accessible, then the class $\mathfrak{X}_{k}$ is accessible for all positive integers $k$.

Proof. Assume for a contradiction that the statement is false, and let $k$ be the smallest positive integer such that there exists an infinite locally graded group $G$ which is not an $\mathfrak{X}_{k}$-group while all its proper subgroups belong to $\mathfrak{X}_{k}$. Clearly, $k>1$ since $\mathfrak{X}$ is accessible. Moreover, $G$ is finitely generated because $\mathfrak{X}_{k}$ is a local class by Lemma 2.1, and hence Lemma 2.3 shows that $G$ is actually soluble-byfinite.

Now [9] implies that the Frattini subgroup $\Phi(G)$ of $G$ has infinite index in $G$. Thus if $N$ is any normal subgroup of finite index in $G$, then there is some maximal subgroup $M$ such that $M N=G$, and hence all finite quotients of $G$ are $\mathfrak{X}_{k}$. Since $\mathfrak{X}_{k}$ is a Robinson class (see Theorem 2.5), we get a contradiction.

It can be immediately checked that both the proofs of Lemma 2.3 and Theorem 2.6 can be used to prove similar results concerning the classes $\overline{\mathfrak{X}}_{k}$; in particular, the analogous of Theorem 2.6 generalizes [7, Theorem 6].

Corollary 2.7. For each positive integer $k$, the class of $k$-quasihamiltonian is accessible.

Lemma 2.8. Let $\mathfrak{X}$ be a local group class which is closed by subgroups, homomorphic images and is made of soluble-by-finite groups. Then every locally graded $\mathfrak{X}_{k}$-group is soluble-by-finite for all $k \geq 1$.

Proof. Assume for a contradiction that the statement is false, and let $k$ be the smallest positive integer such that the class $\mathfrak{X}_{k}$ contains a locally graded group $G$ which is not soluble-by-finite; clearly $k>1$.

If $G^{\prime \prime}$ were not perfect, then $G^{\prime \prime \prime}$ would either have the $\mathfrak{X}_{k-1}$-property, and hence $G$ would be soluble-by-finite by our choice of $k$, or $G / G^{\prime \prime \prime}$ would be quasihamiltonian and hence metabelian, a contradiction. Thus $G^{\prime \prime}=G^{\prime \prime \prime}$.

Let $N$ be any proper normal subgroup of $G^{\prime \prime}$. Then it must be an $\mathfrak{X}_{k-1}$-group; otherwise, $G^{\prime \prime}$ would not be perfect, and by the choice of $k$, it is therefore solubleby-finite. Let $S_{N}$ be the largest normal soluble subgroup of $N$. Then $C_{G^{\prime \prime}}\left(N / S_{N}\right)$ has finite index in $G^{\prime \prime}$, which means that $N / S_{N}$ is abelian, and so $N=S_{N}$ is soluble. 
By Lemma 2.1 and the fact that $G^{\prime \prime}$ is not an $\mathfrak{X}_{k-1}$-group, it follows that there is a finitely generated subgroup $F$ of $G^{\prime \prime}$ not having the $\mathfrak{X}_{k-1}$-property. Since every proper normal subgroup of $G^{\prime \prime}$ is $\mathfrak{X}_{k-1}$, it follows that $F$ is not contained in any proper normal subgroup of $G^{\prime \prime}$ and hence that $G^{\prime \prime}$ contains a maximal proper normal subgroup $S$ (being also the product of two proper normal subgroups of $G^{\prime \prime}$ still proper), which is therefore soluble.

Being simple, $G^{\prime \prime} / S$ does not contain any proper non-trivial permutable subgroup, and this yields that all its proper subgroups have the $\mathfrak{X}_{k-1}$-property. However, Lemma 2.3 shows that $G^{\prime \prime} / S$ is locally graded, and hence it must be either finitely generated or $\mathfrak{X}_{k-1}$ by Lemma 2.1. In any case, the group $G$ is soluble-byfinite.

It was proved in [2] that metaquasihamiltonian groups are soluble with derived length at most 4 . It follows from Lemma 2.8 that locally soluble $\mathfrak{A}_{k}$-groups are soluble, and we can now show that their derived length is bounded in terms of $k$. Here $\mathfrak{Q}^{n}$ denotes the class of soluble groups of derived length at most $n$.

Lemma 2.9. Let $G$ be a soluble group in the class $\left(\mathfrak{U}^{n}\right)_{k}$, where $n$ and $k$ are positive integers. Then $G$ has derived length at most $n+3(k-1)$.

Proof. Working by induction on $k$, we may assume $k \geq 2$. Obviously, if $G^{\prime \prime \prime}$ were not in $\left(\mathfrak{U}^{n}\right)_{k-1}$, then $G^{\prime \prime}=G^{\prime \prime \prime}=\{1\}$ and we would be done. Thus $G^{\prime \prime \prime}$ is in $\left(\mathfrak{Q}^{n}\right)_{k-1}$, and by induction, it is soluble of derived length at most $n+3(k-2)$. It follows that the derived length of $G$ is at most $n+3(k-2)+3=n+3(k-1)$, and the result is proved.

Corollary 2.10. Let $k$ be a positive integer, and let $G$ be a locally soluble $k$-quasihamiltonian group. Then $G$ is soluble of derived length at most $3 k-2$.

The next result shows that a statement similar to that of Lemma 2.3 holds for locally (nilpotent-by-finite) groups, that is, groups whose finitely generated subgroups have a nilpotent subgroup of finite index. It shows in particular that the class of $k$-quasihamiltonian groups locally satisfies the maximal condition on subgroups for all $k \geq 1$.

Lemma 2.11. Let $\mathfrak{X}$ be a class of locally (nilpotent-by-finite) groups. Then all

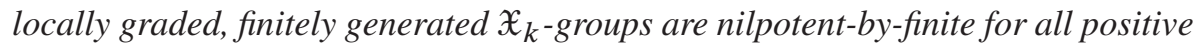
integers $k$.

Proof. Working by induction on $k$, let $G$ be any finitely generated locally graded $\mathfrak{X}_{k}$-group for some $k>1$; clearly $G$ is soluble-by-finite by Lemma 2.3. If all subgroups of $G$ having finite index are permutable, then Robinson's theorem shows 
that $G$ is nilpotent. Thus let $H$ be a subgroup of finite index which is not permutable in $G$. Then $H$ has the $\mathfrak{X}_{k-1}$-property, and it is therefore nilpotent-by-finite, as well as $G$. Induction completes the proof.

Our final result characterizes finite-by-quasihamiltonian groups as those locally graded groups that belong to some $\mathfrak{U}_{k}$. To prove this result, we first deal with periodic and torsion-free cases separately.

Lemma 2.12. Let $G$ be a periodic locally graded $\mathfrak{A}_{k}$ for some positive integer $k$. Then there is a finite normal subgroup $N$ such that the factor group $G / N$ is quasihamiltonian.

Proof. Suppose the result is false, and let $k$ be the smallest positive integer for which there is a periodic locally graded $\mathfrak{A}_{k}$-group $G$ not satisfying the statement; clearly $k>1$. By Lemma 2.1 , there is a finitely generated subgroup $E$ which is not $\mathfrak{A}_{k-1}$, and hence it is permutable in $G$; Lemma 2.8 yields that $E$ is finite.

Let $X$ be any subgroup of $G$; then the product $X E$ is permutable in $G$, and the index $|X E: X|$ is finite. Therefore, every subgroup of $G$ has finite index in a permutable subgroup, and hence $G$ contains a finite normal subgroup $N$ such that $G / N$ is quasihamiltonian (see [3]).

Lemma 2.13. Let $G$ be a torsion-free locally graded $k$-quasihamiltonian group for some positive integer $k$. Then $G$ is abelian.

Proof. Let $k$ be the smallest positive integer for which there is a torsion-free $\mathfrak{A}_{k}$-group $G$ which is not abelian. Then all non-permutable subgroups are abelian so that $G$ is metaquasihamiltonian, and being torsion-free, it is abelian by [2, Lemma 2.5].

Recall now that a soluble-by-finite group $G$ is said to have finite 0-rank $r$ if there is a finite series of subgroups

$$
\{1\}=N_{0} \unlhd N_{1} \unlhd \cdots \unlhd N_{m}=G
$$

such that there are precisely $r$ factors of the series which are infinite cyclic, the others being periodic; if there is no such an integer $r$, then $G$ will be said to have infinite 0-rank. It is clear that, for torsion-free abelian groups, the concept of 0-rank and the usual concept of rank coincide. Recall also that the periodic radical of a group is its largest normal periodic subgroup.

Lemma 2.14. Let $G$ be a locally graded $k$-quasihamiltonian group for some positive integer $k$. Then the set of all periodic elements is a subgroup. 
Proof. Suppose the result is false, and let $k$ be the smallest positive integer such that there exists a counterexample $G$ in $\mathfrak{A}_{k}$; clearly $k \geq 2$. By Lemma $2.11, G$ is locally (nilpotent-by-finite), so we can find two periodic elements $x, y$ in $G$ such that $\langle x, y\rangle$ is not finite and the 0 -rank of $\langle x, y\rangle$ is smallest possible. If $T$ is the periodic radical of $\langle x, y\rangle$, then we can clearly assume $G(=\langle x, y\rangle / T)$ has a normal torsion-free nilpotent subgroup $N$ of finite index, and Lemma 2.13 yields that $N$ is abelian.

If $\left[N^{m}, x\right]=1=\left[N^{n}, y\right]$ for two positive integers $n, m$, then $N^{n m} \leq Z(G)$ so that $G$ would be center-by-finite and hence finite-by-abelian, a contradiction. We may thus assume $\left[N^{m}, x\right] \neq 1$ for all integers $m$. If $N^{m}\langle x\rangle$ belonged to $\mathfrak{A}_{k-1}$ for some $m$, then we would get $\left[N^{m}, x\right]=1$, a contradiction. Therefore, $\langle x\rangle N^{m} / N^{m}$ is permutable in $\langle x\rangle N / N^{m}$ for all $m$, and if we chose $m$ such that $(m, \mathrm{o}(x))=1$, then $\langle x\rangle N / N^{m}$ would even be abelian, being locally nilpotent. As $\langle x\rangle N$ is residually abelian, it is also abelian, the final contradiction.

Now we deal with the case of 0 -rank 1, and in order to do so, the following technical lemma will be very useful, but first define a non-permutability chain of length $n$ in a group $G$ as a chain of subgroups $H_{1}<H_{2}<\cdots<H_{n+1}$ such that $H_{i}$ is not permutable in $H_{i+1}$ for all $i=1, \ldots, n$; it is clear that a group having a non-permutability chain of length $n$ cannot be $n$-quasihamiltonian.

Lemma 2.15. Let $p$ be a prime, and let $G=\langle g\rangle \ltimes H$ be a semidirect product of an infinite cyclic subgroup $\langle g\rangle$ and a quasihamiltonian p-group $H$ with infinite commutator subgroup. Then $G \notin \mathfrak{A}_{\infty}$.

Proof. Suppose $G \in \mathfrak{A}_{k}$ for the minimal possible integer $k$ (clearly $k \geq 2$ ). By the structure of locally finite quasihamiltonian groups, the subgroup $H$ has finite exponent; moreover, there exist an element $h \in H$ and an abelian subgroup $K$ such that $H=K\langle h\rangle$ and $h$ acts like a universal power automorphism on $K$. By our choice of $k$, there is a finitely generated subgroup $F$ which is not $(k-1)$-quasihamiltonian and hence is permutable. Since $G$ locally satisfies the maximal condition, we can assume $F=\left\langle g^{m}\right\rangle S$ for some integer $m$ and some finite subgroup $S$ of $H$. Since $H$ has finite exponent, there exists an infinite subgroup $T$ of $K$ such that $T \cap S=\{1\}$ and $\langle h\rangle T$ has infinite commutator subgroup. Let $t \in T$, and note that, since $F\langle t\rangle=\left\langle g^{m}\right\rangle S\langle t\rangle$ and $S\langle t\rangle$ are subgroups (actually $\langle t\rangle$ is normal in $S\langle t\rangle)$, then $g_{1}=g^{m}$ normalizes $S\langle t\rangle$. But it normalizes $S$, and hence $t^{g_{1}}=s t^{m_{1}}$ for some $s \in S$ and some positive integer $m_{1}$ which is prime to the order of $t$. Now there is a power $g_{2}$ of $g_{1}$ centralizing $S$, and since the exponent of $H$ is finite, it is clear by the above argument that there is a power $g_{3}$ of $g_{2}$ centralizing $T$. Finally, as $G$ locally satisfies the maximal condition on subgroups, there is a power of $g_{3}$ 
centralizing $h$. All we have proved so far allows us to assume $G$ to be of the form $\langle g\rangle \times\langle h\rangle K$.

Let $A$ and $B$ be subgroups of $K$ such that both $\langle h\rangle A$ and $\langle h\rangle B$ have infinite commutator subgroups and $A \cap B=\{1\}=\langle h\rangle \cap A B$.

Take $a$ in $A$ not commuting with $h$. We show that the subgroup

$$
L=\left\langle g^{\mathrm{o}(a)}\right\rangle \times(\langle h\rangle B)
$$

does not permute with $\langle a g\rangle$. Indeed, the only way in which the element

$$
h \cdot a g \in L\langle a g\rangle
$$

could possibly belong to $\langle a g\rangle L$ is by taking a power $a_{1}=a^{m}$ of $a$ such that $a_{1}^{h}=a$ and $m<\mathrm{o}(a)$, and looking at products of the type

$$
a_{1} g^{m+\mathrm{o}(a) \cdot k_{1}} \cdot\left(g^{\mathrm{o}(a)}\right)^{k_{2}} \cdot h \cdot b
$$

for some integers $k_{1}, k_{2}$ and $b \in B$; but $m \neq \equiv 1(\bmod \mathrm{o}(a))$, and so the exponent $m+\mathrm{o}(a) \cdot\left(k_{1}+k_{2}\right)$ of $g$ can never be equal to 1 .

The subgroup $L$ is subject to the same conditions as $G$, and this allows us to construct non-permutability chains of arbitrarily large length, a contradiction proving the statement.

Proposition 2.16. Let $G$ be a locally graded $k$-quasihamiltonian group for some positive integer $k$. If the 0 -rank of $G$ is 1 , then $G$ is finite-by-quasihamiltonian.

Proof. Let $k$ be the smallest positive integer for which there is a counterexample $G$ to the statement; clearly $k \geq 3$. Lemma 2.14 implies that the set of all periodic elements of $G$ is a subgroup, say $T$, while $G / T$ is abelian by Lemma 2.13. Furthermore, there is a finite normal subgroup $N$ of $T$ such that $T / N$ is quasihamiltonian (see Lemma 2.12). In particular, $T / N$ is locally nilpotent, and its primary components are either abelian or of finite exponent. The same can then be said about $T / N_{G}$ so that we may suppose $T$ has those properties, replacing $G$ with $G / N_{G}$ (however, note that quasihamiltonianity could not be preserved).

Our next aim is to show that $T^{\prime}$ is finite. Having this in mind, suppose first that there is some $p$-primary component $T_{p}$ of $T$ with infinite commutator subgroup. Let $g$ be any aperiodic element of $G$, and consider the section $\left\langle g, T_{p}\right\rangle N^{\langle g\rangle} / N^{\langle g\rangle}$ to which an application of Lemma 2.15 gives a contradiction.

Now suppose there are infinitely many non-abelian primary components in $T$, and let $T_{p_{1}}, T_{p_{2}}, \ldots, T_{p_{n}}$ be different non-abelian primary components of $T$ such that $p_{i}$ does not divide $|N|$ for all $i=1, \ldots, n$. Let $g_{n}$ be some aperiodic element, and put $S_{0}=\{1\}, S_{i}=T_{p_{1}} \ldots T_{p_{i}}$ for all $i=1, \ldots, n$. Suppose an aperiodic element $g_{i+1}$ has been defined for some $i$ in $\{0,1, \ldots, n-1\}$. The group 
$\left\langle g_{i+1}\right\rangle S_{i+1} / S_{i}$ contains an infinite cyclic subgroup $\left\langle g_{i+1}^{m_{i+1}} s_{i+1} S_{i}\right\rangle\left(s_{i+1} \in S_{i+1}\right)$ which is not permutable in $\left\langle g_{i+1}\right\rangle S_{i+1} / S_{i}$, and we let $g_{i}=g_{i+1}^{m_{i+1}} s_{i+1}$. In particular, the subgroup $\left\langle g_{i}\right\rangle S_{i}$ is not permutable in $\left\langle g_{i+1}\right\rangle S_{i+1}$. This construction leads to a non-permutability chain of arbitrary length, which contradicts $G$ being a $k$-quasihamiltonian group.

Thus we have reduced so far to the case in which the non-abelian primary components of $T$ have finite commutator subgroup and are finite in number so that we can factor $T^{\prime}$ out and get $T$ abelian.

Let $F=\langle g\rangle S$ be a non-( $k-1)$-quasihamiltonian (and hence permutable) subgroup of $G$, for some $g \in G \backslash T$ and some finite subgroup $S$ of $T$; moreover, write

$$
G=\bigcup_{n \in \mathbb{N}}\left\langle h_{n}\right\rangle T,
$$

where $h_{1}=g$ and $\left\langle h_{n}\right\rangle T \leq\left\langle h_{n+1}\right\rangle T$ for all $n \in \mathbb{N}$. If $n$ is any positive integer, we have $g=h_{n}^{k_{n}} u_{n}$, where $k_{n}$ is an integer and $u_{n} \in T$, so that the subgroup $\left\langle h_{n}^{k_{n}}\right\rangle S$ is permutable in $G$, being isomorphic to $F$. Therefore, $\left\langle h_{n}\right\rangle S$ is a subgroup so that $h_{n}$ normalizes $S$. The arbitrariness of $n$ shows that $S$ is normal in $G$. Being finite, we may assume $S=\{1\}$, and hence all subgroups $\left\langle h_{n}\right\rangle$ are permutable in $G$. In particular, each element of infinite order acts as a power automorphism on $T$, and all elements of prime order and all elements of order 4 of $T$ are central. Theorem 2.4.11 of [13] now implies that $G$ is quasihamiltonian.

Some preliminary work is needed for larger 0-ranks.

Lemma 2.17. Let $G$ be a group and $P$ a normal subgroup of $G$ such that $G / P$ is free abelian of finite 0-rank $r \geq 2$ and $P$ is an abelian p-group of infinite exponent for some prime $p$. If there is some element $h \in G$ acting as an automorphism of infinite order on $P$, then $G \notin \mathfrak{A}_{\infty}$.

Proof. It is enough to show the result in the case $r=2$ and $G=\langle g\rangle\langle h\rangle P$ for some element $g \in G$ of infinite order such that $\langle g\rangle \cap P\langle h\rangle=\{1\}$. Suppose by way of contradiction that $G$ is $k$-quasihamiltonian for some positive integer $k$. Then Proposition 2.16 shows that $P\langle h\rangle$ is finite-by-quasihamiltonian, so there is a finite normal subgroup $N$ such that $P\langle h\rangle / N$ is quasihamiltonian. Factoring out $N^{G}$, which is finite, we reduce ourselves to the case in which $h$ acts like a (locally universal) power automorphism on $P$. In a similar way, we may assume $g$ acting like a (locally universal) power automorphism on $P$. Finally, $h^{g}=h t$ for some $t \in P$, and we even factor out $\langle t\rangle^{G}$ so that $[h, g]=1$.

Let $a$ be an element of $P$ not commuting with $h$, and let $g_{a}$ be a power of $g$ centralizing $a$. Then the subgroup $\left\langle g_{a}^{\mathrm{o}(a)}, h\right\rangle$ does not permute with $\left\langle a g_{a}\right\rangle$ since the element $h \cdot a g_{a} \in\left\langle g_{a}^{\mathrm{o}(a)}, h\right\rangle\left\langle a g_{a}\right\rangle$ does not belong to $\left\langle a g_{a}\right\rangle\left\langle g_{a}^{\mathrm{o}(a)}, h\right\rangle$. 
Let now $a_{n}$ be an element of $P$ having order $(n+1) \cdot \mathrm{o}(a)$ for $n \geq 1$. Then the subgroup $L_{i}=\left\langle g_{a_{n}}^{\mathrm{o}(a)^{i}}, h, a_{n}^{\mathrm{o}(a)^{i}}\right\rangle$ is not permutable in

$$
L_{i-1}=\left\langle g_{a_{n}}^{\mathrm{o}(a)^{i-1}}, h, a_{n}^{\mathrm{o}(a)^{i-1}}\right\rangle
$$

for all $i=1, \ldots, n+1$ as its quotient by $\left\langle a_{n}^{\mathrm{o}(a)^{i}}\right\rangle$ is not such in $L_{i-1} /\left\langle a_{n}^{\mathrm{o}(a)^{i}}\right\rangle$ for the above reasons. Thus we may construct non-permutability chains of arbitrarily large lengths, and this is absurd.

Lemma 2.18. Let $G$ be a locally graded $k$-quasihamiltonian group for some positive integer $k$. Let $N$ be a normal abelian subgroup of $G$ such that the torsion part $T$ of $G$ is contained in $N$ and $G / N$ is locally cyclic and periodic. Suppose also that $\langle z\rangle T$ has a finite commutator subgroup for all aperiodic elements $z$ of $G$. If the 0 -rank of $G$ is finite and $\geq 2$, then $G$ is finite-by-abelian.

Proof. Let $\bar{H}=G / N$, take an aperiodic element $z$ of $N$, and put

$$
\bar{G}=(\bar{H} \ltimes T) \times\langle z\rangle,
$$

with $\bar{H}$ naturally acting onto $T$. It is easy to see that $\bar{G}$ is locally isomorphic to a section of $G$, and hence it must be $k$-quasihamiltonian. Indeed, let $\bar{h}=g N \in \bar{H}$, and take $g_{1} \in G \backslash T$ such that

$$
\langle g\rangle \cap\left\langle g_{1}\right\rangle=\{1\} \quad \text { and } \quad\left[\langle g\rangle T, g_{1}\right]=1,
$$

which is possible by hypothesis and the fact that $G / T$ is abelian; then the section $\left\langle g, g_{1}\right\rangle T /\left\langle g^{\mathrm{o}(\bar{h})}\right\rangle$ is isomorphic to $(\langle\bar{h}\rangle \ltimes T) \times\langle z\rangle$, and the arbitrariness of $\bar{h}$ proves our claim.

Then, by Proposition 2.16 , the group $\bar{G}$ must be finite-by-quasihamiltonian and hence finite-by-abelian. Thus there is a normal subgroup $M$ of $T$ such that $\bar{H} \ltimes T / M$ is abelian so that $T / M$ is central in $G / M$, and we may assume $T$ is central in $G$ factoring out $M$.

Using induction on $k$, we may now assume $G$ is a minimal counterexample with respect to $k$ and $k \geq 2$. Thus there is a finitely generated subgroup $F$ of $G$ which is not $(k-1)$-quasihamiltonian and is such that $G / F T$ is periodic. We factor out the periodic part of $F$ and assume therefore $F$ being torsion-free and permutable in $G$; put $M=T F$, and note that $G / M$ is abelian of finite rank (in the usual sense of abelian groups).

Now let $f \in F$ and $g \in G$ be aperiodic elements such that $[g, f] \neq 1$, in particular $f^{g}=f t$ for some $1 \neq t \in T$. Clearly, $\left|\langle g\rangle: C_{\langle g\rangle}(f)\right|=\mathrm{o}(t)$. The group $\langle g\rangle / C_{\langle g\rangle}(f) \ltimes(T \times\langle f\rangle)$ is isomorphic to a section of $G$ since $T\langle f\rangle \cap\langle g\rangle=1$; otherwise, the subgroup $\langle f, g\rangle$ would be abelian. If $g_{1}$ is another element of $G$ 
such that some power of it is equal to $g m_{1}$ for some $m_{1} \in M$, then $\langle g\rangle / C_{\langle g\rangle}(f)$ embeds in $\left\langle g_{1}\right\rangle / C_{\left\langle g_{1}\right\rangle}(f)$. This shows that the group

$$
G / C_{G}(f) \ltimes(T \times\langle f\rangle),
$$

is locally $k$-quasihamiltonian and hence $k$-quasihamiltonian. Suppose $G / C_{G}(f)$ is infinite. Then, by Proposition 2.16, the above group is finite-by-quasihamiltonian, and $T$ contains elements of the same orders of those contained in $G / C_{G}(f)$. But $T$ is central, and hence this is a contradiction. Thus $G / C_{G}(f)$ is finite, and $f$ has finitely many conjugates in $G$.

It follows that each $f$ in $F$ has finitely many conjugates, and hence it is possible to find a finite subgroup of $T$ such that $T\langle f\rangle$ is normal in $G$. Therefore, there exists a finite subgroup $U$ of $T$ such that $F U / U$ is central in $G / U$. We factor out this subgroup. If $f$ is any element of $G$, then $\langle f\rangle$ is central in $G$ is finite-by-quasihamiltonian. If it is finite-by-abelian, then $G^{\prime}$ would be finite, so, by induction on the 0 -rank, we may assume the 0 -rank of $G$ being 2 .

In this case, suppose first that the commutator subgroup of $G$ has elements of infinitely many pairwise coprime orders, and let $p_{1}, \ldots, p_{n}$ be $n$ of these prime orders. Then there are two infinite cyclic subgroups, say $\langle a\rangle$ and $\langle b\rangle$, such that $a^{b}=a t$ with $\mathrm{o}(t)=p_{1} \cdot \ldots \cdot p_{n}$. Clearly, $T\langle a\rangle \cap\langle b\rangle=\{1\}$, and the quotient

$$
T\langle a, b\rangle /\left\langle a^{\mathrm{o}(t)}, b^{\mathrm{o}(t)}\right\rangle
$$

is a direct product of $n$ non-quasihamiltonian groups, which means it contains a non-permutability chain of length $n$. The arbitrariness of $n$ gives a contradiction.

The index $\left|F^{G}: F\right|$ is finite, but then $G / F^{G}$ is periodic and hence even finiteby-quasihamiltonian. Thus there is a finite normal subgroup $N / F^{G}$ of $G / F^{G}$ such that $G / N$ is quasihamiltonian and periodic. The non-abelian primary components of $G / N$ have finite exponent, which means they are center-by-finite since $G / T F$ has finite rank (in the usual sense of abelian groups) and hence finite commutator subgroup by Schur's theorem. But they are also in a finite number by what we have shown above, and hence $G^{\prime} N / N$ is finite. Therefore, $G^{\prime}$ is finitely generated and even finite.

Before moving on to the next lemma, we recall that, in any group $G$, the set of all elements admitting only finitely many conjugates is a subgroup which is denoted by $\operatorname{FC}(G)$.

Lemma 2.19. Let $G$ be a locally graded m-quasihamiltonian group for some positive integer $m$. If $N$ is any normal abelian subgroup of $G$ such that the torsion part $T$ of $G$ is contained in $N$ and $G / N$ is locally cyclic and periodic, then $T \leq F C(G)$. 
Proof. Suppose that $T \not \subset F C(G)$, and let $x \in T \backslash F C(G)$. For all $n \geq 1$, there are aperiodic elements $g_{1}, \ldots, g_{n} \in G$ such that

$$
\langle x\rangle^{\left\langle g_{1}\right\rangle}<\langle x\rangle^{\left\langle g_{1}, g_{2}\right\rangle}<\cdots<\langle x\rangle^{\left\langle g_{1}, \ldots, g_{n}\right\rangle} .
$$

The hypotheses show that there is $g \in G$ with

$$
\langle x\rangle^{\left\langle g_{1}\right\rangle}=\langle x\rangle^{\left\langle g^{k_{1}}\right\rangle}<\langle x\rangle^{\left\langle g_{1}, g_{2}\right\rangle}=\langle x\rangle^{\left\langle g^{\left.k_{2}\right\rangle}\right.}<\cdots<\langle x\rangle^{\left\langle g_{1}, \ldots, g_{n}\right\rangle}=\langle x\rangle^{\left\langle g^{k_{n}}\right\rangle}
$$

and $k_{n}=1, k_{n-1}\left|k_{n-2}, \ldots, k_{2}\right| k_{1}$. Let $i \in\{1, \ldots, n-1\}$. If $\langle x\rangle^{\left\langle g^{k_{i}}\right\rangle}\left\langle g^{k_{i}}\right\rangle$ permutes with $\left\langle g^{k_{i+1}}\right\rangle$, then $\langle x\rangle^{\left\langle g^{k_{i}}\right\rangle}$ would be normalized by $\left\langle g^{k_{i+1}}\right\rangle$, which is not possible. The arbitrariness of $n$ contradicts the assumption that $G$ is $m$-quasihamiltonian.

We now deal with the case in which the 0-rank is strictly greater than 1 .

Proposition 2.20. Let $G$ be a locally graded $k$-quasihamiltonian group for some positive integer $k$. If the 0 -rank of $G$ is strictly greater than 1 , then $G$ is finite-byquasihamiltonian.

Proof. Let $T$ be the periodic part of $G$, and let $x$ be any element of infinite order. Proposition 2.16 shows that the subgroup $T\langle x\rangle$ is finite-by-quasihamiltonian so that $T$ is finite-by-abelian (see [13, Theorem 2.4.11]), and hence we may even assume $T$ abelian, replacing $G$ by $G / T^{\prime}$.

We now let $k \geq 2$, working by induction on $k$, and let $E$ be a finitely generated subgroup of $G$ which is not $(k-1)$-quasihamiltonian. If $G / T$ has infinite 0 -rank, then there is an element of infinite order modulo $E$ so that $E$ is normal in $G$ by [2, Lemma 2.6]. It follows that $G / E$ is abelian (see [13, Theorem 2.4.11]), which means $G^{\prime}$ is finitely generated and hence even finite, being locally finite. It is therefore possible to consider only the case in which $G / T$ has finite 0 -rank.

Suppose now there is an aperiodic element $g$ not commuting with infinitely many primary components of $T$. Let $T_{p}$ be a $p$-primary component of $T$ such that

$$
\left[T_{p}, g\right] \neq\{1\} .
$$

Lemma 2.17, Proposition 2.16 and the fact that the 0 -rank of $G$ is $\geq 2$ yield the existence of an infinite cyclic subgroup

$$
H=\langle h\rangle \quad \text { with }\left[H, T_{p}\right]=\{1\} \text { and } H \cap T_{p}\langle g\rangle=\{1\} ;
$$

moreover, it is even possible to choose $T_{p}$ such that $[H,\langle g\rangle] \cap T_{p}=\{1\}$. Factoring out $T_{p^{\prime}}$ for the moment being, we obtain $[h, g]=1$. Now $\left\langle g, h^{\mathrm{o}\left(t_{p}\right)}\right\rangle$ does not 
permute with $\left\langle t_{p} h\right\rangle$, where $t_{p}$ is any element of $T_{p}$ not commuting with $g$. We can repeat the process in the subgroup $T_{p^{\prime}}\left\langle g, h^{\mathrm{o}\left(t_{p}\right)}\right\rangle$ getting a contradiction to the fact that $G$ is $\mathfrak{A}_{k}$. This shows that any aperiodic element of $G$ does not commute with finitely many primary components of $T$.

Let $T_{p}$ be any $p$-component of $T$ for some prime $p$, and assume first that it has finite exponent. Let $x \in G$ be an aperiodic element such that $L_{x}=\langle x\rangle T_{p}$ has infinite commutator subgroup. Again, we factor out $T_{p^{\prime}}$ and take $y \in G \backslash T$ with $L_{x} \cap\langle y\rangle=\{1\}$. Since $L_{x}$ is finite-by-quasihamiltonian by Proposition 2.16, there is a finite normal subgroup $N_{x}$ of $L_{x}$ such that $L_{x} / N_{x}$ is quasihamiltonian. Let $C=C_{\langle x\rangle}\left(L_{x} / N_{x}\right)$. Then, applying Lemma 2.15 to $\langle y\rangle L_{x} /\left\langle C, N_{x}\right\rangle^{\langle y, x\rangle}$, we reach a contradiction.

If $T_{p}$ has infinite exponent, then Lemma 2.17 shows that $T_{p}\langle z\rangle$ has finite commutator subgroup for any aperiodic element $z$ of $G$. Summing up, the commutator subgroup of $T\langle z\rangle$ is finite for any $z \in G$.

Now let $F$ be any non- $(k-1)$-quasihamiltonian subgroup of $G$ with maximal 0 -rank. Factoring out the commutator subgroup of the normal subgroup $F T$ (here we use Lemma 2.19 and what we have just proved) makes it possible to apply Lemma 2.18 to each subgroup $L$ of $G$ such that $L / T F$ is periodic and locally cyclic, and hence $L$ is finite-by-abelian. Since $G / T$ has finite rank, we can find a finite normal subgroup $R$ of $G$ such that, factoring it out, we get $T F$ central in $G$.

If the 0-rank of $G$ is strictly greater than 2, by induction on the 0-rank, it follows that $G /\langle f\rangle$ is finite-by-abelian for every non-trivial element $f$ of $F$. Thus $G^{\prime}$ is finitely generated and so finite. We are leaving with the case 0-rank 2 . In this case, suppose first that the commutator subgroup of $G$ has elements of infinitely many pairwise coprime orders, and let $p_{1}, \ldots, p_{n}$ be $n$ of these prime orders. Then there are two infinite cyclic subgroups, say $\langle a\rangle$ and $\langle b\rangle$, such that $a^{b}=a t$ with o $(t)=p_{1} \cdot \ldots \cdot p_{n}$. Clearly, $T\langle a\rangle \cap\langle b\rangle=\{1\}$ and the quotient

$$
T\langle a, b\rangle /\left\langle a^{\mathrm{o}(t)}, b^{\mathrm{o}(t)}\right\rangle
$$

is a direct product of $n$ non-quasihamiltonian groups, which means it contains a non-permutability chain of length $n$. The arbitrariness of $n$ gives a contradiction.

Therefore, $G^{\prime}$ has finitely many non-trivial primary components. Now $G / F$ is quasihamiltonian and periodic so that it contains only finitely many non-abelian primary components. These primary components must have finite exponents, and since $G / T F$ has finite rank (in the usual sense of abelian groups), these nonabelian primary components must even be center-by-finite (recall that $T$ is central) and hence have a finite commutator subgroup by Schur's theorem. Thus $G / F$ has a finite commutator subgroup, which means that $G^{\prime}$ is finitely generated and so finite. 
Theorem 2.21. A locally graded group $G$ is finite-by-quasihamiltonian if and only if it belongs to the class $\mathfrak{A}_{\infty}$.

Proof. If $G$ belongs to the class $\mathfrak{U}_{\infty}$, then Proposition 2.16 and Proposition 2.20 show that it is finite-by-quasihamiltonian.

Conversely, suppose $G$ has a finite normal subgroup $N$ such that $G / N$ is quasihamiltonian. Clearly, there is some non-negative integer $m$ (depending only on the order of $N$ ) such that if $\cdots \leq H_{n+1} \leq H_{n} \leq \cdots \leq H_{1} \leq H_{0}$ is a descending chain of subgroups of $G$ with either $H_{i+1}=H_{i}$ or $H_{i+1}$ not permutable in $H_{i}$, then $H_{j} \cap N=H_{j+1} \cap N$ for all $j \geq m$. Thus

$$
H_{j+1} / N \cap H_{j+1}=H_{j+1} / N \cap H_{j} \leq H_{j} / N \cap H_{j} \simeq H_{j} N / N
$$

for all $j \geq m$. As $H_{j} N / N$ is quasihamiltonian, it follows that $H_{j+1}$ is permutable in $H_{j}$, and hence $H_{j}=H_{j+1}$ for all $j \geq m$. This shows that $H_{m}$ is quasihamiltonian, and hence $G$ is $(m+1)$-quasihamiltonian.

\section{Bibliography}

[1] R. Baer, Überauflösbare Gruppen, Abh. Math. Semin. Univ. Hamburg 23 (1959), $11-28$.

[2] M. De Falco, F. de Giovanni, C. Musella and R. Schmidt, Groups in which every non-abelian subgroup is permutable, Rend. Circ. Mat. Palermo (2) 52 (2003), no. 1, 70-76.

[3] M. De Falco, F. de Giovanni, C. Musella and Y.P. Sysak, Groups in which every subgroup is nearly permutable, Forum Math. 15 (2003), no. 5, 665-677.

[4] F. de Giovanni and M. Trombetti, Large characteristic subgroups with modular subgroup lattice, Arch. Math. (Basel) 111 (2018), no. 2, 123-128.

[5] F. de Giovanni and M. Trombetti, Cohopfian groups and accessible group classes, to appear.

[6] M. R. Dixon, M. Ferrara, Z. Y. Karatas and M. Trombetti, Groups with all subgroups permutable or soluble of finite rank, to appear.

[7] D. Esposito, F. de Giovanni and M. Trombetti, Groups whose non-normal subgroups are metahamiltonian, Bull. Aust. Math. Soc., to appear.

[8] D. Esposito, F. de Giovanni, M. Trombetti, Some trends in the theory of groups with finitely many normalizes, Ric. Mat., to appear.

[9] J. C. Lennox, Finite Frattini factors in finitely generated soluble groups, Proc. Amer. Math. Soc. 41 (1973), 356-360. 
[10] J. C. Lennox and J. S. Wilson, A note on permutable subgroups, Arch. Math. (Basel) 28 (1977), no. 2, 113-116.

[11] D. J.S. Robinson, A theorem on finitely generated hyperabelian groups, Invent. Math. 10 (1970), 38-43.

[12] D. J.S. Robinson, Finiteness Conditions and Generalized Soluble Groups. Part 2, Ergeb. Math. Grenzgeb. (3) 63, Springer, New York, 1972.

[13] R. Schmidt, Subgroup Lattices of Groups, De Gruyter Exp. Math. 14, Walter de Gruyter, Berlin, 1994.

Received October 1, 2019; revised October 20, 2019.

\section{Author information}

Maria Ferrara, Dipartimento di Matematica e Applicazioni "Renato Caccioppoli”, Università degli Studi di Napoli Federico II, Complesso Universitario Monte S. Angelo, Via Cintia, Napoli, Italy.

E-mail: maria.ferrara3@unina.it

Marco Trombetti, Dipartimento di Matematica e Applicazioni "Renato Caccioppoli", Università degli Studi di Napoli Federico II, Complesso Universitario Monte S. Angelo, Via Cintia, Napoli, Italy.

E-mail: marco.trombetti@unina.it 